\title{
MIMO-Aware Routing in Wireless Mesh Networks
}

\author{
Shan Chu and Xin Wang \\ Department of Electrical and Computer Engineering \\ Stony Brook University, Stony Brook, New York 11794 \\ Email: \{schu, xwang\}@ece.sunysb.edu
}

\begin{abstract}
Multiple-input and multiple-output (MIMO) technique is considered as one of the most promising emerging wireless technologies that can significantly improve transmission capacity and reliability in wireless mesh networks. While MIMO has been widely studied for single link transmission scenarios in physical layer as well as from MAC perspective, its impact on network layer, especially its interaction with routing has not drawn enough research attention. In this paper, we investigate the problem of routing in MIMO-based wireless mesh networks. We mathematically formulate the MIMO-enabled multi-source multidestination multi-hop routing problem into a multi-commodity flow problem by identifying the specific opportunities and constraints brought by MIMO transmissions, in order to provide the fundamental basis for MIMO-aware routing design. We then use this formulation to develop a polynomial time approximation solution that maximizes the scaling factor for the concurrent flows in the network. Moreover, we also consider a more practical case where controllers are distributed, and propose a distributed algorithm to minimize the congestion in the network links based on steepest descent framework, which is proved to provide a fixed approximation ratio. The performance of the algorithms is evaluated through simulations and demonstrated to outperform the counterpart strategies without considering MIMO features.
\end{abstract}

\section{INTRODUCTION}

In recent years, uncoordinated multi-hop wireless networks, such as ad hoc and mesh networks have been gaining increased research interest and application. On one hand, these networks are known to provide a number of desirable features, such as easy deployment and maintenance, robustness to node failures and extended coverage. On the other hand, they are expected to serve an increased amount of data traffic with various demands from different users. However, due to the lack of a central controller and the multi-hop, interference limited nature of the network, it is extremely difficult and challenging to meet the higher data traffic requirements. Therefore, it is critical to introduce some new technology that can enable larger network capacity and higher reliability.

Multiple-input multiple-output (MIMO) system has been proven to be able to provide high spectral efficiency and increase channel capacity substantially through multiple spatial channels without need of additional spectrum. With multiple antennas at the transmitter and/or receiver, a MIMO system takes advantage of multiplexing to simultaneously transmit multiple data streams to increase wireless data rate and diversity to optimally combine signals from different transmission streams to increase transmission reliability and range. To meet the high data rate requirements, more and more wireless devices are equipped with multiple antennas. MIMO is prominently regarded as a technology of choice for next generation wireless systems such as IEEE 802.16, IEEE $802.11 \mathrm{n}$, and the third and fourth generation cellular systems. It is also being considered for supporting peer to peer applications over an infrastructure free wireless mesh network.

Most existing studies on applying MIMO technique in ad hoc and mesh networks focus on the physical and MAC layers [1]-[5]. In wireless ad hoc and mesh networks, routing is an important factor that affects the system performance. From a network layer's perspective, MIMO nodes provide different transmission/receiving capabilities from conventional single-antenna nodes. A node equipped with multiple antennas could possibly transmit/receive more downlink/uplink streams, which can significantly impact the determination of optimal routes for traffic transmission. Moreover, the option of different MIMO strategies, i.e. spatial multiplexing or diversity with different levels of degree of freedom, could further increase the flexibility of routing decisions in a network with MIMO nodes. Therefore, it is of paramount importance to have the routing scheme to be MIMO-aware in order to fully leverage the benefits brought by MIMO into wireless networks. Some earlier work, i.e. [6], has made an effort in designing heuristic routing algorithms and protocols. However, to the best of our knowledge, there is very limited work that has studied the problem of routing in MIMO-enabled networks from an optimization perspective and it is still not clear theoretically how much benefit can be achieved by taking advantage of the opportunities and addressing the constraints resulting from the incorporation of MIMO.

In this paper, we study the problem of MIMO-aware routing in wireless mesh networks to leverage the benefits brought by MIMO. Different from previous work, we formally formulate the multi-source multi-destination multi-hop routing problem in MIMO-based wireless mesh networks as a multi-commodity flow problem to model the end-to-end traffic, subject to constraints that model the specific features of MIMO transmissions. We allow more flexible cooperation among nodes. Specifically, nodes in the network can perform many-to-many transmissions, in which a transmitter node can simultaneously transmit to multiple downstream nodes and a receiver node can simultaneously receive from multiple upstream nodes, and a transmission path can be established in reference to different MIMO channel modes based on the statistics of the channel conditions and different traffic demands. With a solid formulation, we provide a sound theoretical upper bound to serves as the reference for a practical system design through an approximated centralized algorithm with polynomial time 
complexity, and we also propose a distributed algorithm with provable efficiency to balance the traffic over the network and control the network congestion.

The rest of the paper is organized as follows. Section II discusses the related work and we introduce the system model in Section III. We define the constraints for MIMO-aware routing and formally formulate the problem in Section IV. Section V presents an alternative formulation of the problem to facilitate a centralized polynomial time approximation solution. Then in Section VI, we propose a distributed approximated solution to solve the joint routing and MIMO channel assignment problem. Finally, we provide the simulation results in Section VII and conclude the paper in Section VIII.

\section{RELATED WORK}

The application of MIMO technique in wireless mesh and ad hoc networks has gained increased attention in recent years. Although a number of efforts have been made in developing efficient MAC schemes to enable MIMO communications in ad hoc networks [1]-[5], there is very limited research on designing routing algorithms and protocols to specifically consider the MIMO features. The authors in [6] propose a routing protocol for ad hoc networks that leverages the various characteristics of MIMO links. A routing algorithm with QoS provisioning is presented in [7] to exploit the multiplexing gain and interference cancelation properties of MIMO antennas. The studies in [6], [7], however, are purely heuristic. In [8], the authors consider the problem of jointly optimizing power and bandwidth allocation at each node and multi-hop/multi-path routing in a MIMO-based ad hoc network. Routing is modeled as a simplified constraint there and the specific features of MIMO transmissions have not been taken into account in establishing routing path. Meanwhile, multi-channel multiradio ad hoc networks have also drawn great research interest [9], [10], [11]. As MIMO system shares some similarity as multi-channel multi-radio system, those works provide a good reference for our design. However, different from fixed frequency channels, MIMO transmission has more modes and there are more complicated constraints to be considered.

\section{System ModeL}

We consider a fixed wireless mesh network where nodes are peers to each other. Nodes in the network are equipped with antenna arrays to facilitate MIMO communications.

With multiple antennas at the transmitter and/or receiver, spatial multiplexing can be used to transmit multiple independent data streams between a node pair. At the receiver, each antenna receives a superposition of all of the data streams. In a rich scattering environment where the transmission channels for different streams are differentiable and independent, i.e., orthogonal, an intended receiver node can separate and decode its received data streams based on their unique spatial signatures. This achieves the multiplexing gain that can provide a linear increase (in the number of antenna elements) in the asymptotic link capacity. As the transmission quality could be very different for multiple spatial paths,

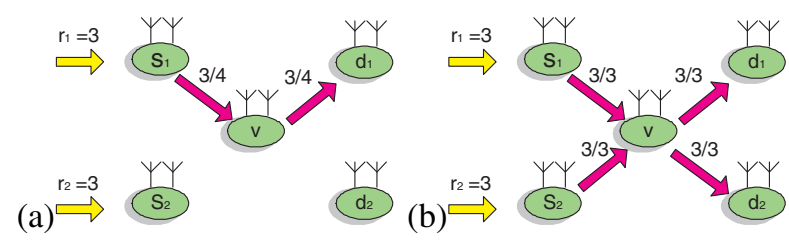

Fig. 1. Illustration of routing in a MIMO-based network.

spatial diversity may be exploited to improve transmission reliability. There are different types of diversity techniques. When channel information is unavailable, dependent streams can be transmitted on different antenna elements over multiple time slots and improve transmission quality through space time coding. With adequate channel information, a subset of antennas that can transmit signals at better quality can be selected for transmissions through selection diversity, which is shown to outperform space-time coding [12].

Although some recent studies have been performed at the physical and MAC layer to address the challenges of leveraging MIMO advantages in networking, we believe that it is very important for the network layer to be aware of the specific characteristics of MIMO nodes and make more intelligent routing decisions. Based on the features of MIMO strategies, the array of antennas in each node can be grouped to form different MIMO channels, with different number of antennas and different achievable channel capacity. Figure 1 illustrates the advantage of using MIMO-aware routing. In a network of five nodes, node $s_{1}$ and $s_{2}$ have 3 units of traffic demand for $d_{1}$ and $d_{2}$ respectively. The end-to-end paths of the two traffic flows both have to go via node $v$. As each node is equipped with two antennas in this network, we assume that the channel using two antennas has the capacity 4 and the channel using one antenna has the capacity 3 for each link. As node $v$ can receive two independent data streams at most, a conventional routing strategy can route at most 3 units of traffic for either $s_{1}$ or $s_{2}$ as shown in Figure 1 (a), using the 2-antenna channel. As a better alternative, MIMO-aware routing can adaptively select the set of MIMO-channels to route the traffic, so that all the 6 units of traffic demand can be satisfied as in Figure 1 (b), by having each link use the 1-antenna channel.

Due to the specific features of MIMO-based network, we have the following issues to consider in the design.

- Link capacity. Although in conventional networks the link capacity generally depends on network topology and channel conditions, in MIMO-based networks, it also depends on the size of the antenna arrays of nodes. For a transmission link between a node pair, the link capacity can be chosen from a set of varied capacities of different antenna combinations and strategies. Moreover, more than one combination may be used simultaneously to form several MIMO channels. The actual capacity of each MIMO channel can be estimated on a periodic basis and the statistics is used in routing decision.

- Link channel assignment. As an antenna array has limited size, the number of simultaneously used antenna combinations should not exceed the available number of 
antennas of the node, which is known as transmitter degree constraint in scheduling. Meanwhile, for simultaneous transmissions from multiple spatial channels, the set of antennas used by different spatial channels should not overlap, which we name it as antenna compatibility constraint. Also, as different antenna combinations have different capacities, it is important to determine which antenna combination to use when a route is determined.

- Interference consideration. With multiple antennas, a node can receive data streams while canceling interference streams concurrently, and the total number of received streams depends on the antenna size, which is described as receiver degree constraint in scheduling. From the perspective of routing, the antenna size can be also regarded as a measure of a node's capability of concurrent data receiving and interference cancellation.

- Multi-path routing. As nodes are endowed with manyto-many transmission capability by multiple antennas, it is beneficial to incorporate multi-path routing for end-toend flows in order to better exploit multi-path diversity and maximize throughput. While using multi-path may lead to the problems regarding packet re-ordering and loss recovery, these issues have been studied in literature work on multi-path routing and are beyond the scope of this paper.

In this paper, we focus on routing traffic between different source/destination pairs and corresponding MIMO mode selection. The problem of scheduling the flow in a specific time slot is beyond the scope of this paper.

\section{PRoblem Formulation}

Based on the system model described in Section III, we formally formulate the MIMO-aware routing problem in wireless mesh networks as an optimization problem. In order to model end-to-end traffic, we use a multi-commodity flow model for the routing of data packets across the network. That is, source nodes may send different data to their intended destination nodes through multi-path and multi-hop routing.

\section{A. Graph representation}

We represent the multi-hop wireless network via node topology graph $G=\left(V, E \bigcup E^{I}, F\right)$, where $V$ is the set of nodes in the network, $F$ is the set of data flows to be routed, $E$ is the set of directed edges between nodes that can transmit data from one to the other and $E^{I}$ is the set of directed edges which indicate the interference from a transmitter to nodes within its interference range during data transmission. To be more specific, given a data link $e \in E, t(e) / h(e)$ is used to represent the transmission/receiving end of the link $e$, and there is a directed edge from $t(e)$ to $h(e)$. In the network, there is a set of sources $\mathbf{s}$, which send data to a set of destinations $\mathbf{d}$, with the end-to-end rate demand vector r. Assume the rate vector has $F<|V|(|V|-1)$ components. Each source-destination pair between which there is a traffic request is termed as a commodity. Let $s(f) / d(f)$ represent the source/destination node for commodity $f$, and $r(f)$ represent the flow that has to be routed from $s(f)$ to $d(f)$.

\section{B. Channel and flow constraints}

Define the concept MIMO channel $(\mathcal{M C})$ as the MIMO spatial channel over a link that uses a designated set of antennas and corresponds to a specific MIMO operation mode. Denote the set of MIMO channels over link $e$ as $\mathcal{M C}(e)$, and each element $\mathcal{M C}_{i}(e) \in \mathcal{M C}(e)$ has a size, denoted as $m_{i}^{t}(e)$ for the transmitter node and $m_{i}^{h}(e)$ for the receiver node, which is the number of antennas used for constructing this MIMO channel at the two ends of the link $e$. By using different sizes, a set of MIMO channels can be constructed to take advantage of spatial multiplexing and/or spatial diversity. Note that, with the calculation of antenna weights at transmitters and transmit over eigen-modes of the channel, MIMO channels can be considered orthogonal [13]. Suppose node $v \in V$ has $N_{v}^{a n t}$ antennas. Each MIMO channel $(e, i)$ is associated with a set of antennas of node $t(e)$, which is indicated using the parameter $u_{i, a, e}\left(a=1, \ldots, N_{t(e)}^{a n t}\right) . u_{i, a, e}=1$ if and only if the MIMO channel $i$ over link $e$ uses antenna $a$ of node $t(e)$ for transmission. For example, as in Figure 1, node $v$ has two antennas $a_{0}$ and $a_{1}$, which can be used to compose different MIMO channels for the link $e=v \rightarrow d_{1}$. Channels 0 and 1, i.e. $(e, 0)$ and $(e, 1)$, both use one antenna, so $m_{0}^{t}(e)=$ $m_{1}^{t}(e)=m_{0}^{h}(e)=m_{1}^{h}(e)=1$, and thus $u_{0,0, e}=u_{1,1, e}=1$ and $u_{0,1, e}=u_{1,0, e}=0$. Channel 2 and 3, i.e. $(e, 2)$ and $(e, 3)$, are constructed by transmitting simultaneously over antennas $a_{0}$ and $a_{1}$, so $u_{2,0, e}=u_{2,1, e}=u_{3,0, e}=u_{3,1, e}=1$ and $m_{2}^{t}(e)=m_{3}^{t}(e)=2$. If the MIMO transmission strategy used for channel 2 is spatial multiplexing, i.e., independent data streams are transmitted simultaneously from the two antennas, the receiver has to use at least two antennas for successful decoding, therefore $m_{2}^{h}(e)=2$. Alternatively, if space-time coding, i.e. Alamouti code [14], is used for the transmission over channel 3 , the receiver only needs one antenna for decoding so $m_{3}^{h}(e)=1$. Note that the values of $m_{i}^{h}(e), m_{i}^{t}(e)$ and $u_{i, a, e}$ are easy to obtain off-line and are static for each node.

Each data link $e$ has capacity $c_{i}(e)$ on MIMO channel $i$, and there is an estimated capacity for a given MIMO channel over a link for an estimation period. The set of $\mathcal{M C} s$ and the values of $c_{i}(e)$ can be saved as a look-up table and updated in each estimation period according to the topology/channel condition variations. The length of the period should be properly determined so that the value of $c_{i}(e)$ can correctly reflect the actual link condition. We use $x_{i}^{f}(e)$ to denote the flow on channel $i$ over data link $e$ that carries the data of the end-to-end flow session $f$, and define $g_{i}(e)=\sum_{f} x_{i}^{f}(e) / c_{i}(e)$ as the utilization of MIMO channel $i$ over link $e$ for all flows.

A necessary condition for rate vector $\mathbf{r}$ to be achievable is the existence of link flow $x_{i}^{f}(e)$ that satisfies the following flow conservation constraints: 


$$
\begin{array}{r}
\sum_{e: t(e)=s(f)} \sum_{i \in \mathcal{M C}(e)} x_{i}^{f}(e)=r(f), \forall f ; \\
\sum_{e: h(e)=d(f)} \sum_{i \in \mathcal{M C}(e)} x_{i}^{f}(e)=r(f), \forall f ; \\
\sum_{e \in E_{\text {in }}(v)} \sum_{i \in \mathcal{M C}(e)} x_{i}^{f}(e)=\sum_{\substack{e \in E_{\text {out }}(v) \\
\forall f, \forall v \neq \mathcal{M}(e)}} x_{i}^{f}(e), \\
\forall f(f), d(f) ;
\end{array}
$$

where $E_{\text {in }}(v)$ and $E_{\text {out }}(v)$ are incoming and outgoing edges of node $v$ in the set $E$. As the flow capacity constraint, each link should satisfy:

$$
\sum_{f} x_{i}^{f}(e) \leq c_{i}(e), \forall e, \forall i \in \mathcal{M C}(e),
$$

which can be simplified as $g_{i}(e) \leq 1, \forall e, i \in \mathcal{M C}(e)$.

While constraints (1)-(4) are conventional for flow problems, the use of MIMO technique imposes new constraints. Even though the degree constraints introduced in Section III are generally formulated in MAC layer, they actually have a significant impact over routing in the network layer. In order to address these constraints, we first present them with linkflow variables in each time slot, and then translate them into end-to-end rate variables for routing purpose.

Let $I_{e, i, \tau}$ be the indicator variable that has value 1 if and only if channel $i$ is active over link $e$ at time slot $\tau$. Note that the channels over outgoing edges of $v$ in $E$ are considered active if there are data transmissions from node $v$, and the channels over incoming edges of $v$ in the set $E$ and $E^{I}$ are considered active if there are data transmissions and interference transmissions to $v$ respectively. To satisfy the degree constraint at the transmitter side, the number of antennas used by the active outgoing edges of a node $v$ must be no larger than its number of antennas $N_{v}^{a n t}$ in each time slot $\tau$ :

$$
\sum_{e \in E_{\text {out }}(v)} \sum_{i \in \mathcal{M C}(e)} m_{i}^{t}(e) I_{e, i, \tau} \leq N_{v}^{a n t}, \forall v .
$$

Similarly, corresponding to the receiver's degree constraint, the total number of antennas that is required to decode the receiving transmissions, including data and interference transmissions, should not exceed the receiving capability of the node. Therefore, we have:

$$
\sum_{e \in E_{i n}(v) \cup E_{i n}^{I}(v)} \sum_{i \in \mathcal{M C}(e)} m_{i}^{h}(e) I_{e, i, \tau} \leq N_{v}^{a n t}, \forall v .
$$

Suppose routing is performed for each $T$ time slots. Adding these sets of equations for all the $T$ time slots and dividing by $T$ results in the constraints:

$$
\begin{aligned}
\sum_{e \in E_{\text {out }}(v)} \sum_{i \in \mathcal{M C}(e)} m_{i}^{t}(e) g_{i}(e) & \leq N_{v}^{\text {ant }}, \forall v \\
\sum_{e \in E_{i n}(v) \cup E_{\text {in }}^{I}(v)} \sum_{i \in \mathcal{M C}(e)} m_{i}^{h}(e) g_{i}(e) & \leq N_{v}^{a n t}, \forall v
\end{aligned}
$$

where $g_{i}(e)$ is the fractional link utilization for channel $i$ over link $e$. Specifically, $g_{i}(e)=\sum_{f} \frac{x_{i}^{f}(e)}{c_{i}(e)}=\frac{1}{T} \sum_{1 \leq \tau \leq T} I_{e, i, \tau}$ for all $e$ and $i$.

In addition, each node only has a limited number of antennas, and an antenna cannot be used for transmission over different MIMO channels simultaneously. To address this antenna compatibility constraint, we use the indicator variable $u_{i, a, e}$ introduced earlier to represent the constraint as follows:

$$
\sum_{e \in E_{\text {out }}(v)} \sum_{i \in \mathcal{M C}(e)} u_{i, a, e} I_{e, i, \tau} \leq 1, \forall \tau, v, a .
$$

Similarly as in (7)(8), adding these sets of equations for all the $T$ time slots and dividing by $T$, we have:

$$
\sum_{e \in E_{\text {out }}(v)} \sum_{i \in \mathcal{M C}(e)} u_{i, a, e} g_{i}(e) \leq 1, \forall v, a .
$$

\section{Optimization formulation}

So far, we have derived the set of constraints for a feasible flow for routing data packets in a MIMO-based mesh network. There are many different objectives of interest that can be solved using an optimization framework. Based on the constraints, we formulate the routing problem in the form of a concurrent flow problem, where the desired rate vector is scaled and the objective is to determine the maximum scaling factor $\lambda$ that satisfies the necessary conditions. In this way, the fairness in the resource allocation over flows can be ensured. The resulting linear program (LP) is given below:

$$
\max \lambda,
$$

Subject to:

$$
\begin{aligned}
& \sum_{e: t(e)=s(f)} \sum_{i \in \mathcal{M C}(e)} x_{i}^{f}(e)=\lambda r(f), \forall f ; \\
& \sum_{e: h(e)=d(f)} \sum_{i \in \mathcal{M C}(e)} x_{i}^{f}(e)=\lambda r(f), \forall f ; \\
& \sum_{e \in E_{\text {in }}(v)} \sum_{i \in \mathcal{M C}(e)} x_{i}^{f}(e)=\sum_{e \in E_{\text {out }}(v)} \sum_{i \in \mathcal{M C}(e)} x_{i}^{f}(e), \\
& \forall f, \forall v \neq s(f), d(f) ; \\
& \sum_{e \in E_{\text {out }}(v)} \sum_{i \in \mathcal{M C}(e)} m_{i}^{t}(e) g_{i}(e) \leq N_{v}^{a n t}, \forall v ; \\
& \sum_{e \in E_{i n}(v) \cup E_{i n}^{I}(v)} \sum_{i \in \mathcal{M C}(e)} m_{i}^{h}(e) g_{i}(e) \leq N_{v}^{a n t}, \forall v ; \\
& \sum_{e \in E_{\text {out }}(v)} \sum_{i \in \mathcal{M C}(e)} u_{i, a, e} g_{i}(e) \leq 1, \forall v, a ; \\
& 0 \leq g_{i}(e) \leq 1, x_{i}^{f}(e) \geq 0, \forall e, i \in \mathcal{M C}(e) ;
\end{aligned}
$$

where equations (12)-(14) are flow conservation constraints, equations (15)-(16) stand for the routing constraints as the result of using MIMO antenna arrays, and equation (17) is used to meet the antenna compatibility constraint.

So far, we have presented a straightforward formulation with flow variables. 


\section{The Centralized Algorithm}

The optimization problem formulated in section IV is linear and can generally be solved by linear optimization algorithms, i.e. simplex method. However, the complexity is still an important concern. In this section, we follow the work in [15] and [9], and develop a fully polynomial time approximation algorithm using primal-dual algorithm, which is simple to implement and therefore can be potentially applied in a practical wireless network. In order to facilitate the solution, we first reformulate the problem using edge-path formulation and generalize the constraints, which is amenable to the development of the algorithm, then we describe the primal-dual algorithm to solve the optimization problem and obtain the maximum scaling factor $\lambda$.

\section{A. Edge-path reformulation}

First, note that the set of constraints (15)-(17) share a similar format in that each of them concerns a specific set of link/MIMO-channel pairs, so it is possible to generalize them into a simpler form for easier reformulation. Suppose there are $\mathcal{L}$ sets $\left\{Q_{j}\right\}$ composed of link/MIMO-channel pairs that are as defined in constraints (15)-(17), then each of these constraints can be stated in the form as follows:

$$
\sum_{(e, i) \in Q_{j}} \alpha_{i}(e) g_{i}(e) \leq \beta\left(Q_{j}\right), j=1,2, \ldots, \mathcal{L},
$$

where $\alpha_{i}(e)$ and $\beta\left(Q_{j}\right)$ are constants associated with the above constraints. For example, for a node $v^{*}$, constraint (15) concerns the set $Q_{j}=\left\{(e, i) \mid e \in E_{\text {out }}\left(v^{*}\right) \& i \in \mathcal{M C}(e)\right\}$, the corresponding constants are then $\alpha_{i}(e)=\left\{m_{i}^{t}(e) \mid e \in\right.$ $\left.E_{\text {out }}\left(v^{*}\right) \& i \in \mathcal{M C}(e)\right\}$ and $\beta\left(Q_{j}\right)=N_{v^{*}}^{a n t}$. In this way, although the number of constraints as described in (15)-(17) remains the same, they are generalized into a single formula (19).

In order to have an approximate solution, we first reformulate the problem into an edge-path formulation, so that the multi-commodity flows are represented as positive LPs. Let $\mathcal{P}_{f}$ represents the set of all possible simple paths composed of link/MIMO-channel pairs for the commodity $f$. For a path $P \in \mathcal{P}_{f}$ that is from $s(f)$ to $d(f)$, let $x(P)$ be the amount of flow on this path, constraints (12)-(14) are then translated to:

$$
\sum_{P \in \mathcal{P}_{f}} x(P)=\lambda r(f), \forall f .
$$

Furthermore, $x_{i}(e)$, the total amount of flow on channel $i$ over link $e$ is given by:

$$
x_{i}(e)=\sum_{f} \sum_{P \in \mathcal{P}_{f},(e, i) \in P} x(P), \forall(e, i) .
$$

As $g_{i}(e)=x_{i}(e) / c_{i}(e)$, equation (19) becomes:

$$
\sum_{(e, i) \in Q_{j}} \alpha_{i}(e) \frac{\sum_{f} \sum_{P \in \mathcal{P}_{f},(e, i) \in P} x(P)}{c_{i}(e)} \leq \beta\left(Q_{j}\right), j=1, \ldots, \mathcal{L} .
$$

In this constraint, link/MIMO-channel pairs that are both on path $P$ and in set $Q_{j}$, i.e. $(e, i) \in$
$P \cap Q_{j}$, are examined. Consider a single path $P$, from (22), we have $\sum_{(e, i) \in P \cap Q_{j}} \frac{x(P)}{c_{i}(e) / \alpha_{i}(e)} \leq$ $\sum_{(e, i) \in Q_{j}} \alpha_{i}(e) \frac{\sum_{f} \sum_{P \in \mathcal{P}_{f},(e, i) \in P} x(P)}{c_{i}(e)} \leq \beta\left(Q_{j}\right)$. Therefore, $x(P, j)=\beta\left(Q_{j}\right)\left(\sum_{(e, i) \in P \cap Q_{j}} \frac{1}{c_{i}(e) / \alpha_{i}(e)}\right)^{-1}$ is the maximum amount of flow on path $P$ allowed by $Q(j)$.

In summary, the edge-path formulation of the constraints in the original optimization problem is restated as follows:

$$
\begin{array}{r}
\sum_{(e, i) \in Q_{j}} \frac{\sum_{f} \sum_{P \in \mathcal{P}_{f},(e, i) \in P} x(P)}{\beta\left(Q_{j}\right) c_{i}(e) / \alpha_{i}(e)} \leq 1, \\
j=1,2, \ldots, \mathcal{L} ; \\
\sum_{P \in \mathcal{P}_{f}} x(P)=\lambda r(f), \forall f ; \\
x(P) \geq 0, \forall P \in \mathcal{P}_{f}, \forall f .
\end{array}
$$

\section{B. Primal-dual solution}

According to the weak duality property, the objective value of any feasible solution of the minimization problem gives an upper bound on the optimal objective of the dual maximization problem. Following [15] and [9], we formulate the dual of the LP problem and develop a fully polynomial time approximation algorithm using a primal-dual algorithm. Let $y(j)$ be the dual variables for each set $Q_{j}$, and $z(f)$ be the dual variable for the rate scaling constraints in (25). The dual of the LP problem is then as follows:

Subject to:

$$
\min \sum_{j} y(j)
$$

$$
\begin{array}{r}
\sum_{(e, i) \in P} \frac{\sum_{j:(e, i) \in Q_{j}} \frac{\alpha_{i}(e) y(j)}{\beta\left(Q_{j}\right)}}{c_{i}(e)} \geq z(f), \forall P \in \mathcal{P}_{f}, \forall f ; \\
\sum_{f} r(f) z(f) \geq 1 ; \\
y(j) \geq 0, j=1, \ldots, \mathcal{L} .
\end{array}
$$

The dual problem is essentially an assignment of lengths to link/MIMO-channel pairs, such that $\sum_{j} y(j)$ is minimized. The proposed primal-dual algorithm is given in algorithm 1 .

The algorithm initially assigns a weight of $\delta$ to all sets $Q_{j}$, and then proceeds in phases. In each phase we route $r(f)$ units of flow from $s(f)$ to $d(f)$ for each commodity $f$, and a phase ends when all the $F$ commodities are routed. For each commodity $f$, The $r(f)$ units of flow from $s(f)$ to $d(f)$ are sent via multiple iterations, as in lines 5-13. In each iteration, each pair $(e, i)$ is assigned with a length $\frac{\sum_{j:(e, i) \in Q_{j}} y(j) / \beta\left(Q_{j}\right)}{c_{i}(e) / \alpha_{i}(e)}$, and a corresponding shortest path path $P^{*}$ from $s(f)$ to $d(f)$ that minimizes the sum of the length is determined by a shortest path algorithm, i.e., the Dijkstra's algorithm. Among the sets in $\left\{Q_{j}\right\}$ that have the intersection with $P^{*}$, we compare their values of maximum allowable flow $x\left(P^{*}, j\right)$, and the one with the minimum value $u=\min _{j:(e, i) \in P^{*} \&(e, i) \in Q_{j}} x\left(P^{*}, j\right)$ is the amount of the flow that can be sent on $P^{*}$ in this iteration. Moreover, since $r(f)$ units of flow have to be sent for commodity $f$ in each phase, the actual amount of flow sent is the lesser of $u$ and the remaining amount of flow $r$ to 


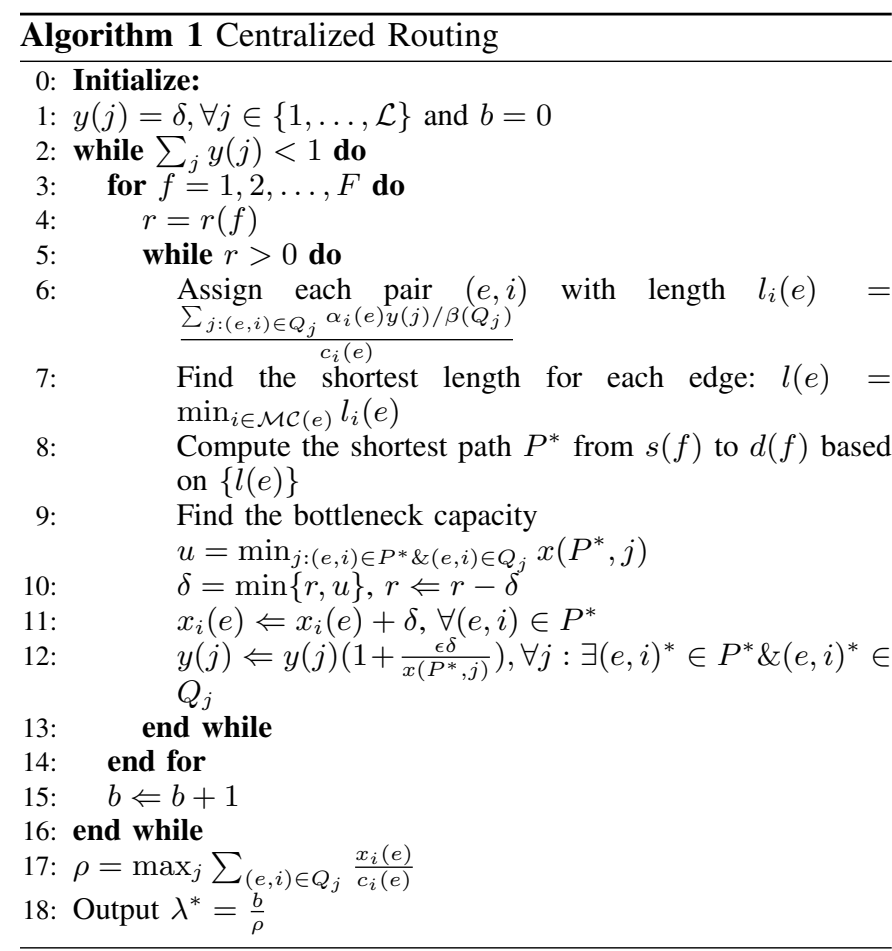

make up $r(f)$ in this phase. Once a flow is sent via a path, the weights of the sets $\left\{Q_{j}\right\}$ associated with the link/MIMOchannel pairs that carry the flow is updated, as in line 12 . The algorithm then alternates between sending flow along shortest paths and adjusting the length of the link/MIMO-channel pairs along which flow has been sent until an optimal solution is reached.

The complexity of the primal dual algorithm mainly lies in solving a sequence of shortest path problems. Following [15], it can be shown that by choosing $\delta$ and $\epsilon$ appropriately, the solution can get as close to the optimum solution as desired at the expense of increasing running time, as in the following remark.

Remark I: The algorithm 1 computes a $(1-\epsilon)^{-3}$ optimal solution to the scaling factor of the maximum concurrent flow problem in time polynomial in $F, \mathcal{L},|V|$ and $1 / \epsilon$, where $F$ is the number of commodities, $\mathcal{L}$ is the number of constraining sets, and $|V|$ is the number of nodes.

\section{The Distributed Algorithm}

The primal-dual algorithm in the previous section gives an upper bound on the achievable maximum concurrent throughput. In many practical wireless mesh networks, it is important to develop a distributed algorithm, where the computing of routes is performed in a distributed manner to approach a global optimization objective. It is therefore more practical to use an alternative objective function, i.e. to optimally distribute the end-to-end traffic into different paths and link/channel pairs thus balance the load and control the congestion of the network.

In this section, we follow [16] and derive a distributed version of the MIMO-aware routing algorithm in wireless mesh networks that can achieve fast convergence to the near-optimum solution. We assume that each commodity is associated with an agent. The multiple agents make parallel routing decisions without coordination with each other. The only accessible global information for each agent is a common clock and the utilization level of the network edges. The objective is to route $r(f)$ amount of flow for flow $f$ from $s(f)$ to $d(f)$ for all $f \in F$, possibly along several paths, such that the maximum ratio of the total flow routed along an link/MIMO-channel pair $(e, i)$ to its capacity is minimized. In other words, we aim to distribute the traffic evenly in the network and hopefully no $(e, i)$ would be congested or overloaded. Recall that the utilization of a MIMO-channel over an edge $e$ is previously defined as $g_{i}(e)=\sum_{f} \frac{x_{i}^{f}(e)}{c_{i}(e)}$, and the objective is to minimize $\max _{(e, i)} g_{i}(e)$. The distributed scheduling scheme is described in Algorithm 2. Throughout the algorithm, $x_{i}^{f}(e)$ and $l_{i}^{f}(e)$ are the current flow value and length of $(e, i)$ for the agent of commodity $f$ respectively, and $x^{f}$ is the amount of flow that has been routed in the current phase for commodity $f$.

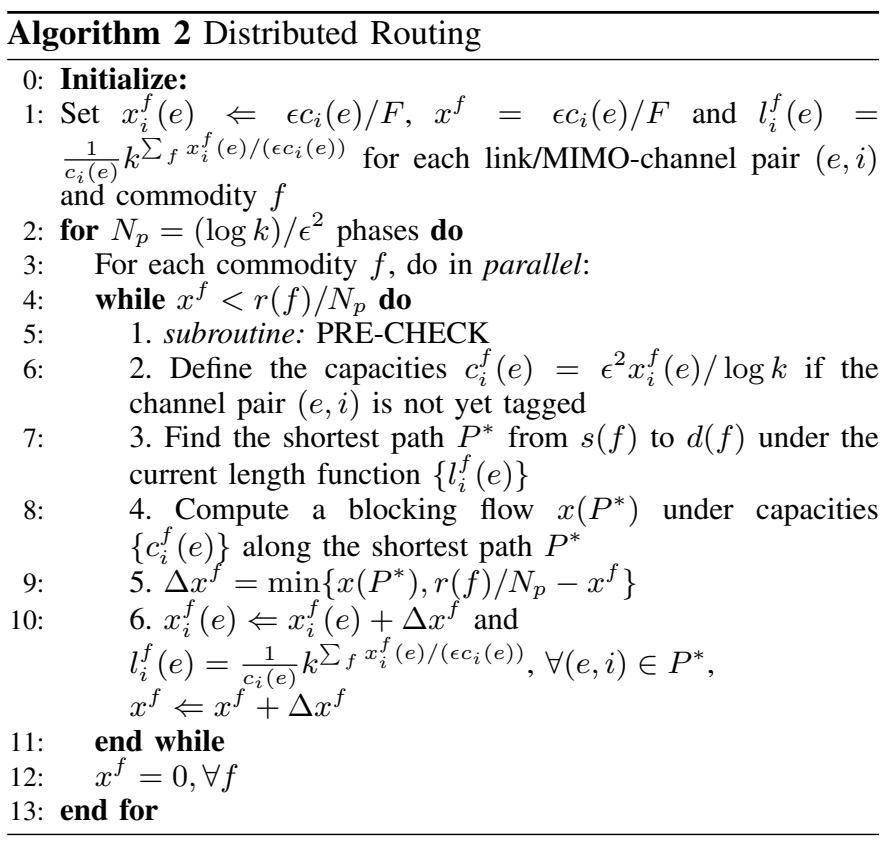

Similar to the centralized algorithm, the distributed algorithm is also based on the steepest descent framework as in [15]. Let $k$ be the number of $(e, i)$ pairs in the network, obviously $k \sim O(|E|)$. The algorithm goes though $N_{p}=(\log k) / \epsilon^{2}$ phases. A flow of amount $r(f) / N_{p}$ is routed for each commodity $f$ in each phase and a feasible solution is derived at the end. For each phase, the process is further divided into steps, as in the while loop in line 411. In each step, each commodity performs in parallel to route a fraction of its own flow. Different from the case in the centralized algorithm, we have the additional problem of how to efficiently perform concurrent routing in a distributed scenario, as concurrent attempts to route on a shortest path may lead the path to be no longer shortest and result in 


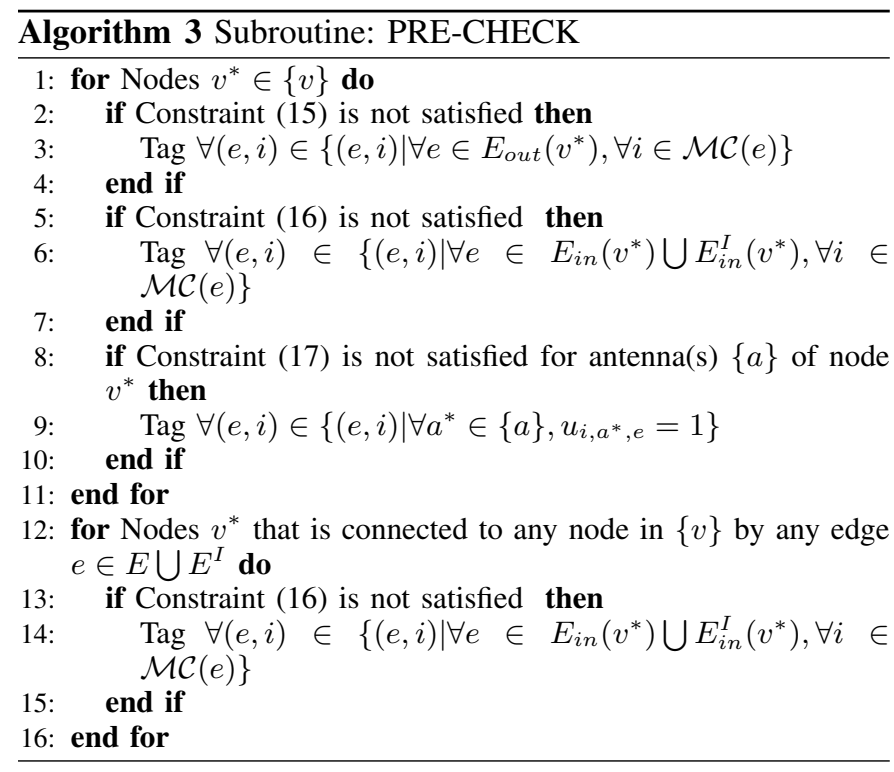

unpredictable oscillations. To handle this problem, a special approach is to guarantee the so-called step-size constraint, that the length increase of any link/channel pair $(e, i)$ can be no larger than an $\epsilon$ fraction. Throughout the algorithm, we initially route a tiny amount of flow of all commodities on all link/MIMO-channel pairs, and later increase the flow multiplicatively. Although the initial pre-flow may not even satisfy the flow conservation constraints, as its total capacity is $\epsilon$ of the actual capacities, it has effect on the optimality only to the extent $\epsilon$. In each step, the algorithm computes the shortest path based on the current length function, and determines the blocking flow along the path, which is the maximum amount of flow that can be routed in the path under the capacity constraint $c_{i}^{f}(e)$ for each $(e, i)$. By computing the blocking flow, it saturates at least one edge on the path, which effectively reduces the number of steps.

In order to make the solution feasible, especially for MIMObased networks, we revisited the constraints in equations (15)(17). Denote $\{v\}$ as the set of nodes that are in the augmenting paths of the previous step. Recall that $g_{i}(e)=\sum_{f} \frac{x_{i}^{f}(e)}{c_{i}(e)}$ can be regarded as a measure of congestion of channel $i$ over link $e$. Therefore, a PRE-CHECK step is added at the beginning of each step, as in algorithm 3, so that each node that is included in the augmenting paths of the last step examines if it still satisfies constraints (15)-(17). If either (15) or (16) is not satisfied, the node can no longer accept extra load, so its incident edges are set to have capacity 0 for all the possible MIMO channels. If (17) is not satisfied, it indicates that some of the antennas, say $a$, of the node is fully-loaded, so MIMO channels that have $u_{i, a, e}=1$ are set to have capacity 0 . We also check the nodes that are connected to nodes in the augmenting paths by edges in $E \cup E^{I}$, in order to account for the interference from the flows in the augmenting paths.

We first prove that the algorithm can achieve a $(1+O(\epsilon))$ approximation. The analysis proceeds as in [16], but is slightly different since a link can be associated with several MIMO channels in our algorithm. Denote $\Phi$ as the potential of the network:

$$
\Phi=\sum_{(e, i)}\left(k^{1 / \epsilon}\right)^{g_{i}(e)} .
$$

Assume the optimum value of $\max _{(e, i)} g_{i}(e)$ is 1 , so the optimum value of $\Phi$ satisfies $\Phi^{*} \geq k^{1 / \epsilon}$.

Consider phase $p$ and step $t$, let $l^{f}(t)$ and $l^{f}(t)^{\prime}$ be the length of the shortest path at the beginning and the end of the step for commodity $f$. In each step, each commodity simultaneously augments its flow along certain paths. Suppose commodity $f$ augments flow $\Delta x_{i}^{f}(e)$ along $(e, i)$, the total additional flow is $\Delta x_{i}(e)=\sum_{f} \Delta x_{i}^{f}(e)$. We first calculate the overall increase in $\Phi$ due to the augmentation along $(e, i)$ :

$$
\begin{array}{r}
\Delta \Phi(e)=k^{x_{i}(e) / \epsilon c_{i}(e)}\left(k^{\left.\Delta x_{i}(e) / \epsilon c_{i}(e)-1\right)}\right. \\
\leq k^{\frac{x_{i}(e)+\Delta x_{i}(e)}{\epsilon c_{i}(e)}} \cdot \frac{\Delta x_{i}(e) \log k}{\epsilon c_{i}(e)} \\
=\sum_{f} \Delta x_{i}^{f}(e) \frac{\log k}{\epsilon} \frac{k^{g_{i}(e)^{\prime} / \epsilon}}{c_{i}(e)},
\end{array}
$$

where $g_{i}(e)^{\prime}$ is the utilization factor after the augmentation step. The inequation (32) is derived from the inequality $e^{a}-$ $1 \leq a e^{a}$ by letting $a=\frac{\Delta x_{i}(e) \log k}{\epsilon c_{i}(e)}$. Note that $\frac{1}{c_{i}(e)} k^{g_{i}(e)^{\prime} / \epsilon}$ is the length of $(e, i)$ in the next step, denoted as $l_{i}^{f}(e)^{\prime}$. Based on the above inequation, the total increase in $\Phi$ at the end of this step is:

$$
\begin{array}{r}
\Delta \Phi \leq \sum_{(e, i) \in P^{*}} \sum_{f} \Delta x_{i}^{f}(e) \frac{\log k}{\epsilon} l_{i}^{f}(e)^{\prime} \\
=\frac{\log k}{\epsilon} \sum_{f} \Delta x^{f} l^{f}(t)^{\prime} \\
\leq \frac{\log k}{\epsilon} \sum_{f} \Delta x^{f}(1+\epsilon) l^{f}(t),
\end{array}
$$

where $P^{*}$ is the shortest path found on line 7 of Algorithm 2, Inequation (34) is derived directly from (33), equation (35) is derived from the fact that the blocking flow values on all edges of the path $P^{*}$ are the same and equal to $\Delta x^{f}$, and $l^{f}(t)^{\prime}=\sum_{(e, i) \in P^{*}} l_{i}^{f}(e)^{\prime}$ is the length of the shortest path. Inequation (36) is from the step-size constraint which ensures that the length increase of each edge can be at most an $\epsilon$ fraction of the original length, i.e. $l_{i}^{f}(e)^{\prime} \leq(1+\epsilon) l_{i}^{f}(e)$. Note that $l^{f}(t) \leq l^{f}(p)$ where $l^{f}(p)$ is the length of the shortest path at the end of phase $p$. As we route $r(f) / N_{p}=\epsilon^{2} r(f) / \log k$ amount of flow for each commodity $f$ in each phase, we can estimate the change in the potential during phase $p$ as follows:

$$
\begin{array}{r}
\Phi(p)-\Phi(p-1) \leq \frac{\log k}{\epsilon} \sum_{f} \sum_{t} \Delta x^{f}(t)(1+\epsilon) l^{f}(t) \\
\leq \epsilon(1+\epsilon) \sum_{f} r(f) l^{f}(p) .
\end{array}
$$

Denote the optimum solution to the problem as $\left\{x_{i}^{f}(e)^{*}\right\}$. Note that $l_{i}^{f}(e)$ for all $f$ is the same at the end of phase $p$, denoted as $\left.l_{i}(e)\right|_{p}$. For each $(e, i)$, since $\sum_{f} x_{i}^{f}(e)^{*} \leq c_{i}(e)$, we have: 
(a)

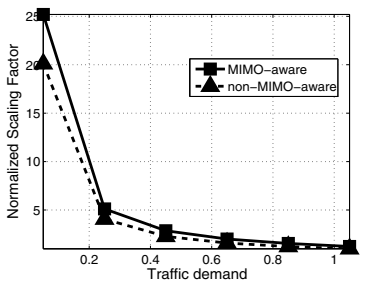

(b)

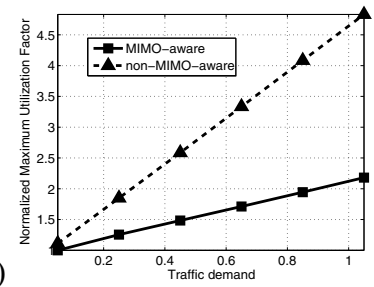

Fig. 2. Grid topology: impact of traffic demand.

(a)

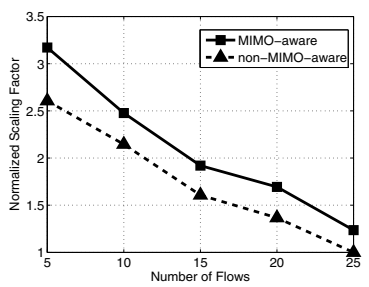

(b)

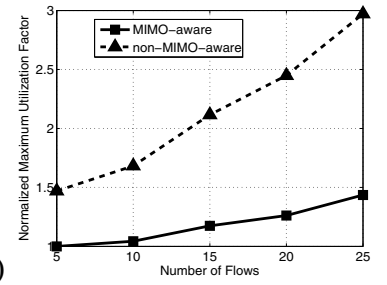

(a)

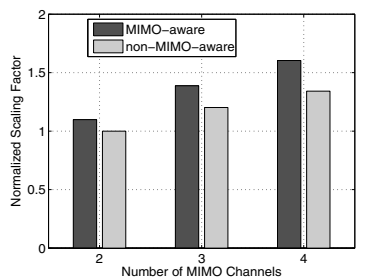

(b)

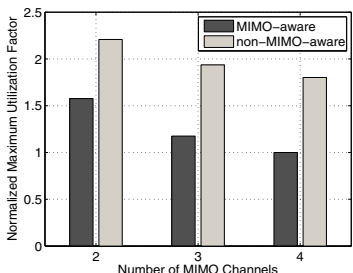

Fig. 4. Grid topology: impact of the number of MIMO channels.

(a)

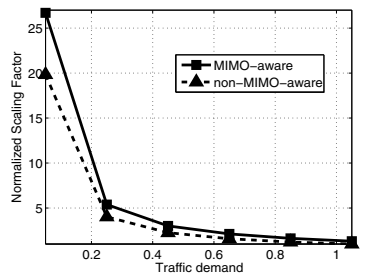

(b)

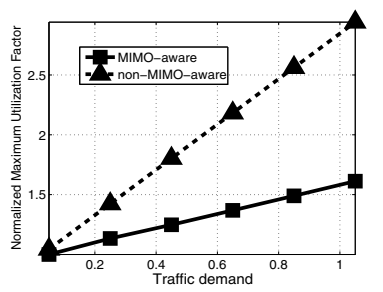

Fig. 3. Grid topology: impact of the number of flows.

$\Phi(p)=\left.\sum_{(e, i)} c_{i}(e) l_{i}(e)\right|_{p} \geq\left.\sum_{f} \sum_{(e, i)} x_{i}^{f}(e)^{*} l_{i}(e)\right|_{p} \geq \sum_{f} r(f) l^{f}(p)$,

as $l^{f}(p)$ is the shortest path length from $s(f)$ to $d(f)$ and the total flow amount is $r(f)$. Combining (38) and (39), we have $\Phi(p) \leq \Phi(p-1) /(1-\epsilon(1+\epsilon))$. Initially, $g_{i}(e)=0$ for all $(e, i)$, so $\Phi(0)=k$. As $N_{p}=\log k / \epsilon^{2}$, we have $\Phi\left(N_{p}\right) \leq k\left(\frac{1}{1-\epsilon+\epsilon^{2}}\right)^{\log k / \epsilon^{2}} \leq k^{O(1)} k^{1 / \epsilon} \leq k^{O(1)} \Phi^{*}$. It can be proved that an $k^{O(1)}$-approximation of $\Phi$ yields a $(1+O(\epsilon))$ approximation of $\max _{(e, i)} g_{i}(e)$.

Once the algorithm runs to the end, we can get the solution with $\left\{x_{i}^{f}(e)\right\}$, which actually includes the end-to-end routes and the corresponding link/MIMO-channel pairs for each flow commodity. From [16], it can be shown that the convergence time of the proposed algorithm is bounded and essentially linear in the maximum path length of the network. We then arrive at the following remark.

Remark II: The distributed algorithm achieves an $1+O(\epsilon)$ approximation to the optimum solution. The while loop can ends in $O\left(L\left(\log ^{2} k \log (F / \epsilon)\right) / \epsilon^{4}\right)$ steps, where $L$ is the largest number of edges in a path.

\section{ViI. Performance Evaluation}

In this section, the performance of our proposed algorithms is evaluated through simulations. Our goal is to verify that by adaptively selecting a set of MIMO channels for each link subject to MIMO constraints, the MIMO-aware routing can achieve better performance under different network settings, compared with the reference non-MIMO-aware routing strategy which does not have the flexibility to switch between channels and always uses the MIMO-channel with the highest capacity for each link. The evaluated performance metrics are the objectives of the proposed algorithms, namely the scaling factor $\lambda$ (which is also a measure of achievable throughput) for
Fig. 5. Random topology: impact of traffic demand.

the centralized algorithm and the maximum utilization factor $g^{*}=\max _{(e, i)} g_{i}(e)$ for the distributed algorithm.

We generate both grid and random topologies, and run simulations with different parameter settings. In each evaluated network, a node is equipped with an array of antennas to facilitate MIMO transmission. For a link with $N_{t}^{\text {ant }} / N_{h}^{\text {ant }}$ antennas at transmitter/receiver ends, we consider up to $\widetilde{N}=$ $\min \left\{N_{t}^{a n t}, N_{h}^{a n t}\right\}$ MIMO channels are available to the link, with each MIMO channel corresponding to one MIMO operation mode and having the degree-of-freedom value ranging from 1 to $\widetilde{N}$. The channel capacity value is estimated by averaging over a sequence of fading coefficients and set as an empirical parameter for each topology setting. We generally assume all the nodes have the same number of antennas $N^{\text {ant }}$ to show the performance improvement by MIMO-aware routing, and we also present the performance under different values of $N^{\text {ant }}$. The traffic in the network is modeled by two parameters: the number of flows $F$ and the demand of each flow $r(f)$. For simplicity, all flows are assumed to have the same demand, whose value is normalized to the capacity of MIMO-channel with the degree-of-freedom 1. The constants $\delta$ and $\epsilon$ used in the algorithms are set as empirically derived values. The default values of $N^{a n t}, F$ and $r(f)$ are 4,15 and 0.5 respectively, and the network has 30 nodes if not otherwise specified. For the clarity of comparison, results are normalized with regard to the minimum value in each figure.

We first study the performance in a grid topology. Consider a $5 \times 6$ grid topology with 30 nodes and each node has at most 4 neighbors. Dividing the grid into four quadrants and the four nodes centered in each quadrant are set as sinks for flows. The destination of a node is the sink node that is closest to it. As traffic demand increases in figure 2, MIMO-aware routing consistently obtains a larger value of scaling factor $\lambda$ (up to $25 \%$ higher) and a smaller maximum utilization factor $g^{*}$ (up to $55 \%$ lower) than that for non-MIMO-aware routing. With an increased number of flows, MIMO-aware routing improves 
(a)

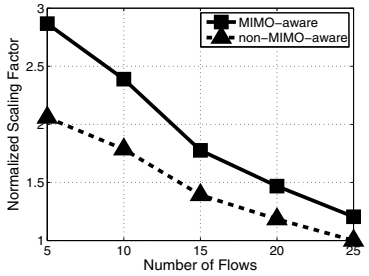

(b)

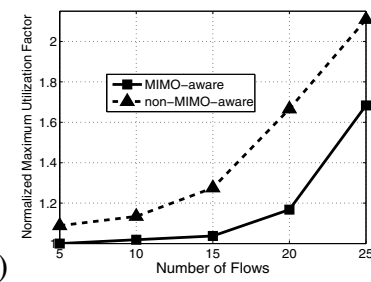

Fig. 6. Random topology: impact of the number of flows.

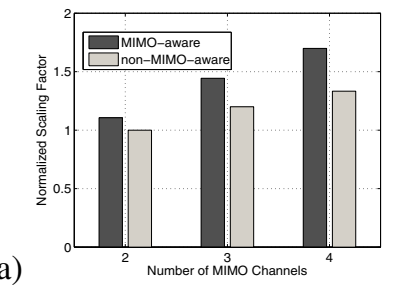

(b)

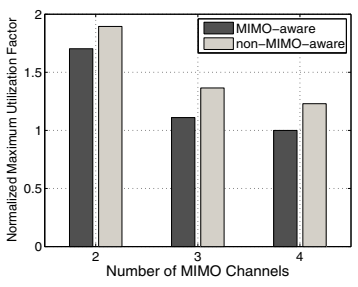

Fig. 7. Random topology: impact of the number of MIMO channels.

$\lambda$ up to $25 \%$ and reduces $g^{*}$ up to $50 \%$ as in figure 3 . The results show that by being aware of the MIMO constraints and adaptively selecting MIMO channels, the traffic demand in the network can be better served. We can further observe from figure 4 that the advantage of MIMO-aware routing is even more significant with the increase of the number of MIMOchannels, as the improvement of $\lambda$ and $g^{*}$ increases from $10 \%$ to $22 \%$ and $32 \%$ to $45 \%$ respectively, when the number of MIMO channels in each link increases from 2 to 4 . With more MIMO channels, there are more options for performing more flexible routing.

Figures 5-7 show the performance of our routing algorithms in random topologies. A random topology is generated by populating nodes randomly in a $500 \times 500$ grid. The transmission range is set as 100 , and each topology generated is ensured to be connected. For each flow, the source and destination are randomly selected from the set of nodes in the network. Each data point is obtained by averaging over 20 different random topologies. In figure 5, a $33 \%$ increase in $\lambda$ and a $45 \%$ decrease in $g^{*}$ are achieved with increasing traffic demand. As the number of flows in the network increases in figure 6, MIMO-aware routing outperforms its counterpart by up to $45 \%$ higher $\lambda$ and $32 \%$ lower $g^{*}$. The results in random topologies are consistent with that in the grid topology and demonstrate that being MIMO-aware is an effective way to leverage MIMO benefits and improve routing performance. The better performance also exists for different number of MIMO-channels, as in figure 7.

\section{CONCLUSIONS}

As a promising technology to improve transmission capacity and reliability in wireless mesh networks, MIMO has been studied extensively in physical and MAC layers, but has not drawn much attention from network layer's perspective. In this paper, we propose the concept of MIMO-aware routing and investigate how it can further leverage the advantages brought by MIMO. We first present constraints that capture the characteristics of MIMO transmissions, and mathematically formulate the MIMO-enabled multi-source multi-destination multi-hop routing problem into a multi-commodity flow problem. We then propose a centralized algorithm to provide an approximated solution to achieve maximum concurrent flow in the network, as well as a distributed algorithm that minimizes the maximum congestion of link/MIMO-channels. The performance of our algorithms is evaluated through simulations with varied traffic demands, number of flows, and available MIMO channels. The results demonstrate that our MIMOaware routing algorithms significantly outperform the routing scheme that does not consider MIMO transmission features and constraints in all the test scenarios. The results in this paper provide a basis for our future work on practical MIMOaware routing protocol design.

\section{REFERENCES}

[1] M. Hu and J. Zhang. MIMO ad hoc networks: Medium access control, saturation throughput, and optimal hop distance. Journal of Communications and Networks, Special Issue on Mobile Ad Hoc Networks, pages 317-330, 2004.

[2] M Zorzi, J Zeidler, A. Anderson, B. Rao, J. Proakis, A.L. Swindlehurst, M. Jensen, and S. Krishnamurthy. Cross-layer issues in MAC protocol design for MIMO ad hoc networks. IEEE Wireless Communication Magzine, (4):62-76, August 2006.

[3] K. Sundaresan, R. Sivakumar, M. Ingram, and T-Y. Chang. A fair medium access control protocol for ad-hoc networks with MIMO links. In Proc. IEEE INFOCOM 2004, June 2004.

[4] S. Chu and X. Wang. Opportunistic and cooperative spatial multiplexing in MIMO ad hoc networks. In Proc. ACM MobiHoc 2008, pages 63-72, May 2008.

[5] S. Chu and X. Wang. Adaptive and distributed scheduling in heterogeneous MIMO-based ad hoc networks. In Proc. IEEE MASS 2009, pages 217-226, Oct 2009

[6] K. Sundaresan and R. Sivakumar. Routing in ad-hoc networks with MIMO links. In Proc. IEEE ICNP 2005, pages 85-98, 2005.

[7] A. Gkelias, F. Boccardi, C.H. Liu, and K.K. Leung. MIMO routing with QoS provisioning. In Proc. ISWPC 2008.

[8] J. Liu, Y.T. Hou, Y. Shi, and H. Sherali. Cross-layer optimization for mimo-based wireless ad hoc networks: Routing, power allocation, and bandwidth allocation. IEEE J. Select. Areas of Commun., (6):913-926, August 2008.

[9] M. Kodialam and N. Thyaga. Characterizing the capacity region in multi-radio multi-channel wireless mesh networks. In Proc. ACM Mobicom '05, pages 73-87, May 2005.

[10] M. Alicherry, R. Bhatia, and L. Li. Joint channel assignment and routing for throughput optimization in multi-radio wireless mesh networks. In Proc. ACM Mobicom '05, pages 58-72, May 2005.

[11] X. Lin and S. Rasool. A distributed joint channel-assignment, scheduling and routing algorithm for multi-channel ad-hoc wireless networks. In Proc. IEEE Infocom '07, pages 58-72, May 2007.

[12] Y. Pan and S. Aissa. Performance analysis of selective space-time coding and selection diversity under perfect and imperfect CSI. In Proc. PIMRC 2005, pages 2371-2375, September 2005.

[13] D. Tse and P. Viswanath. Fundamentals of Wireless Communication. Cambridge University Press, May 2005.

[14] S. M. Alamouti. A simple transmit diversity technique for wireless communications. IEEE J. Select. Areas Commun., pages 1451-1458, October 1998.

[15] N. Garg and J. Koenemann. Faster and simpler algorithms for multicommodity flow and other fractional packing problems. In Proc. FOCS '98.

[16] B. Awerbuch, R. Khandekar, and S. Rao. Distributed algorithms for multicommodity flow problems via approximate steepest descent framework. In Proc. SODA '07. 